\title{
Limit Theorems for Markets with Sequential Bargaining*
}

\author{
Douglas Gale \\ University of Pennsylvania, Philadelphia, Pennsyluania 19104 \\ and University of Pittsburgh, Pittsburgh. Pennsylvania 15260
}

Received September 11, 1985; revised July 21, 1986

\begin{abstract}
This paper models trade as a non-coopertative, strategic game played at an infinite sequence of dates. A single, indivisible commodity is traded. Buyers and sellers have transferable utility and are characterized by their reservation utilities. They meet at random and "bargain" over the price at which a single unit of the good will be exchanged. Under a variety of circumstances it is shown that as the costs of search and bargaining become negligible, the outcome of the game converges to the competitve (flow) equilibrium, even when there is complete information. Journal of Economic Literature Classification Numbers: 022. i. 1987

Academic Press, Inc.
\end{abstract}

\section{INTRODUCTION}

Stock markets, commodity futures markets, and a few other well-known types of markets are characterized by centralized trading. In these markets, traders gather in a single location and all have access to the same trading opportunities. But there are really very few markets in which trading can be described as completely centralized. In many more, trading is decentralized. Yet economists tend to ignore these institutional differences and apply the Walrasian model of competitive equilibrium, even in decentralized markets, if certain general conditions are met. The conditions themselves are familiar enough. There must be a large number of individually insignificant agents. Agents must have symmetric information about the commodities being traded. There must be no transaction costs. And so on. As a convenient shorthand, markets satisfying these conditions are referred to as frictionless. The conventional wisdom, then, is that frictionless markets are competitive and the conventional practice is to use Walrasian models to analyse them. It is an interesting question whether the

* I would like to thank Karl Shell and an anonymous referee for suggesting numerous improvements in the exposition of the paper. Research support was provided by CARESS and the Sloan Foundation. 
Walrasian equilibrium is a good approximation to what goes on in all frictionless markets.

This question has been given a sharper point by recent work which suggests that, in models of decentralized trade, the outcome is not Walrasian even when all frictions are removed. The literature on decentralized trade includes work by Diamond $[3,4]$, Mortensen $[9,10]$, Rubinstein-Wolinsky (RW) [13], Binmore-Herrero [1, 2], Shaked Sutton [14], and Mandel [8]. What all these contributions have in common is the assumption that trade is conducted between pairs of agents. These agents are assumed to meet at random and bargain over the terms on which they will trade. Diamond and Mortensen preceded the others but their analysis was, in onc important respect, ad hoc. They assumed that when a pair of agents met, the division of the gains from trade was governed by the Nash Bargaining Solution. The justification for this assumption is not obvious and one might reasonably suspect that it alone accounted for the non-Walrasian outcome. A more convincing argument was put forward by $\mathrm{RW}$, who were the first to highlight this paradoxical result. They adopted a strategic approach. They modeled decentralized trade as a non-cooperative game and analysed the perfect equilibria of the game. Since their analysis is crucial to an understanding of everything that follows, it is worth sketching the outlines of it here.

One interpretation of the RW model, though not the only one, is that it represents the market for an indivisible commodity. Trade takes place at an infinite sequence of dates. At each date there is a large number of identical buyers and identical sellers in the market. Each seller has one unit to dispose of; each buyer wants to buy at most one unit. Buyers and sellers are randomly matched in pairs at each date. In any given period an agent either will meet no one or will meet exactly one agent of the opposite type. RW take these matching probabilities to be primitives of the model. In most economic applications, however, these probabilities will be endogenous. And in the application of their model to the analysis of a market RW make the probabilities a function of the numbers of agents of each type. If there are more buyers than sellers, for example, a buyer's probability of being matched is less than a seller's

Buyers and sellers remain in the market until they have traded. Then they leave the market. At any date the number of successful buyers leaving the market is necessarily equal to the number of sellers. In order to maintain a stationary state, in which matching probabilities are constant over time, equal numbers of buyers and sellers must enter the market at each date. The numbers of buyers and sellers in the market, by contrast, are constant but unequal in a stationary state.

All agents are assumed to have transferable utilities. Since all buyers and all sellers are identical there is no loss of generality in normalizing the gains 
from trade between buyer and seller to equal unity. When a buyer and seller meet they face the classical bargaining problem: how to divide this unit-sized cake. This problem has been solved in a two-person framework by Rubenstein [11]. His theory is easily extended to the present case where agents meet at random. Consider a buyer and seller who have just met. One of them is chosen at random to be the proposer; the other automatically becomes the responder. The proposer makes a proposal as to how the cake should be divided. The responder responds by accepting or rejecting the proposal. If he accepts, the proposal is implemented immediately. The agents exchange a single unit of the commodity, divide the surplus in the agreed manner and leave the market. If he rejects the proposal both agents are required to remain passive until the next date. $\Lambda t$ the next date, before bargaining can resume, one or both of the agents may meet a new partner. If this happens, the newly matched agent will abandon his old partner in favor of the new one. If neither agent has been matched with a new partner they are free to resume bargaining. In this way, agents bargain and search until at last they reach agreement with an agent of the opposite type and leave the market.

There is one crucial assumption which has not yet been mentioned. Search and bargaining are costly. More precisely, agents are assumed to discount futurc utilitics. The longer it takes them to reach agrecment the smaller, other things being equal, their final payoffs will be. This cost represents an important source of friction in the market and one should not expect a Walrasian outcome as long as there is positive discounting. The relevant question is what happens as the rate of time preference approaches zero.

The exogenous matching process and the rules governing pair-wise bargaining together define a non-cooperative game in extensive form. ${ }^{1}$ The appropriate solution concept is the (subgame) perfect equilibrium. RW make one further restriction: they require the perfect equilibrium to be quasi-stationary. That is, the population of agents in the market is constant over time, their strategies do not depend on time in an essential way and they are independent of history in the sense that an agent's action at a given date depends only on his current state and not on what happened in previous periods. RW show that when there is positive discounting there is

\footnotetext{
'Strictly speaking, the Rubinstein-Wolinsky framework does not constitute an "extensive form." For example, their game has no initial nodes. There seems to be no danger in using the term "extensive form" to describe their setup, however, since these details hardly obtrude into the analysis. Similarly, it is slightly inaccurate to use the term "subgame perfect equilibrium" to describe the solution concept. The reason is that strategies are not defined for certain possible but economically irrelevant nodes of the game. Again, there seems to be no real harm in using the term "subgame perfect equilibrium" in this context and I think most readers will find it more descriptive of what is going on than the available alternatives.
} 
a unique, quasi-stationary, perfect equilibrium. The agents' payoffs are determined by their relative impatience and the matching probabilities. More precisely, it is the ratio of their respective rates of time preference which determines the payoff, not the absolute levels. Allowing their respective rates of time preference to converge to zero and holding the ratio constant does not change their relative shares.

This result is the basis of RW's critique of the competitive paradigm. Here is a model which satisfies all the requirements of a frictionless market. $\Lambda$ ccording to the conventional wisdom, the outcome of any reasonable theory of price formation should, under these conditions, be the Walrasian equilibrium. And yet, they observe, this is patently not the case. A Walrasian equilibrium requires that if there are more sellers than buyers the price should fall to zero. Conversely if there are more buyers than sellers the price should equal unity. In short, the agents on the long side of the market should receive a zero payoff. But the outcome of the bargaining game will always assign a positive payoff to buyers and sellers as long as the ratio of their rates of time preference is positive and finite.

Other examples of non-Walrasian equilibria in frictionless markets have been given by Shaked-Sutton [15] and Mandel [8]. In the present paper, however, I am only concerned with the result of RW.

The RW paradox does not arise in all models of exchange. In [6] I studied a general, exchange economy and showed that a perfect equilibrium of the corresponding bargaining game always implements a Walrasian equilibrium of the underlying economy. (An earlier version [5] introduced the analytical methods on which [7] and the present paper are based. The conclusion of that paper was more or less the same as [6] but applied to steady-state equilibria only). There are three major differences between the model I studied in [6] and the one used by RW. ${ }^{2}$ First, there is no discounting. Rather than assuming some positive degree of discounting and then taking limits, I chose to work directly with the limiting economy. Second, commodities are assumed to be divisible. As a result there is no reason why an agent should not trade with many other agents. In the RW model sketched above the existence of a single indivisible commodity means that each agent can trade only once. Third, instead of considering a stationary state I took the flow of agents into the economy to be fixed but arbitrary, subject to the important qualification that the total measure of agents entering the market be finite. This last condition has the important implication that the set of all agents entering the market con-

\footnotetext{
${ }^{2}$ In addition to the differences arising from the definition of the model I should mention two assumptions required for the proof of the theorem. The first imposed certain regularity conditions on utility functions (smoothness, concavity, etc.). The second required that at each date the distribution of agents' types should have a diffuse support. It now appears that this last assumption, though needed for the proof given in [7], is not necessary for the result.
} 
stitutes a well-defined exchange economy. We can therefore talk about the Walrasian equilibria of that economy. In a stationary state, with a constant, positive measure of agents entering at each date, the set of all agents necessarily has infinite measure. One cannot speak of a Walrasian equilibrium for the economy consisting of all these agents. Instead, RW and others consider the Walrasian equilibria of the hypothetical economy comprising just the agents in the economy at a single date. This, as we shall see, is not at all the same thing.

Apart from these differences, the spirit of the model presented in [6] was the same as the one presented by RW. In particular, the rules of the bargaining game were the same $\mathrm{e}^{3}$ once allowance was made for the fact that [6] treats a general, exchange economy. So now we have two, apparently contradictory, results. On the one hand, we have a rather general model of decentralized exchange whose perfect equilibria are all Walrasian. On the other hand, we have several small models whose perfect equilibria are nonWalrasian even in the limit as the frictions generated by discounting become small. The central, unresolved issue is what accounts for the difference between these cases and, following on that, how robust is each of them?

There are three possible answers to this question, corresponding to the three principal differences between the models used in [6] and in [7]. First, it is possible that a limit theorem gives a different answer from a theorem "in the limit." In other words, there is a discontinuity at the point where the rate of time preference equals zero. Second, it may be that the indivisibilities in the non-Walrasian models are at the root of the paradox. Third, it could be the difference between stationary state models with an infinite measure of agents and non-stationary models with a finite measure of agents which accounts for the non-Walrasian outcome. In order to resolve the central issue we need to analyze all three possibilities. That analysis is the substance of this paper.

The model I use is a generalization of the RW model. It represents a market for a single commodity. Once again agents are of two types, buyers or sellers, but agents may be distinguished by their reservation or limit prices. If all sellers (resp. buyers) are assumed to have the same reservation price (resp. limit price) then the model collapses to the RW model. At the other extreme, by having a large number of finely graduated reservation or limit prices one can approximate arbitrarily closely a market with continuous demand and supply curves. Thus, even though each agent wishes

\footnotetext{
${ }^{3}$ Rubinstein [13] has suggested that the bargaining in [7] has a "take it or leave it" character which is different from the sequential bargaining in RW. In [7] it is assumed that agents are rematched each period, so that at most a single offer passes between two agents before they separate. A careful examination of the proofs, however, reveals that this assumption is not necessary for the results; it mainly serves to simplify the notation.
} 
to trade a single unit of the indivisible commodity, in the aggregate the market looks like one in which a perfectly divisible commodity is traded. In between these extremes one has a situation like that pictured in Fig. 1 below. The step functions are demand and supply curves. Vertical distances represent the limit price or reservation price of the marginal agent at that point. Horizontal distances represent the measure of agents with that limit price or reservation price.

The first result I establish for this model is a limit theorem for an economy with a finite measure of agents. These agents are assumed to enter the economy according to some fixed but arbitrary pattern. Since their measure is finite the set of all such agents constitutes a well-defined cxchange cconomy. This cconomy generically has a unique Walrasian equilibrium price and a unique Walras allocation. All agents are assumed to discount future utilities at the same rate. (This is a simplifying assumption which is not cssential for the ccntral conclusions of the paper.) Allowing the common discount rate to converge to zero, we generate a sequence of perfect equilibria and their corresponding allocations. A limit point of this sequence of perfect equilibrium allocations is shown to be a Walras allocation of the underlying exchange economy. Binmore and Herrero [2] have also studied non-steady state equilibrium, using the basic RW model. They assume that two types of agents bargain over a unit surplus. All agents are in the market at the first date. They show that in the limit, as the discount rate converges to zero, all the surplus goes to the type of agent that is present in smaller numbers. Interpreting the model as a market for an indivisible commodity, this is the competitive outcome. The price is zero (resp. one) if there are more sellers than buyers (resp. buyers

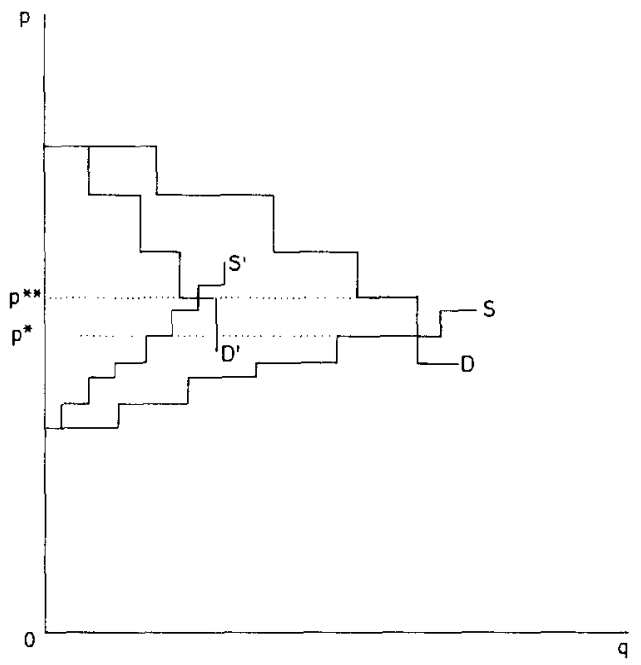

FigURE 1 
than sellers). Their analysis admits the case where two agents may bargain over several periods. (In my model, agents are re-matched every period, though this does not appear to be crucial to the central conclusions). They introduce the concept of security equilibrium, which proves to be a powerful analytical tool, to deal with these more difficult cases.

The competitive result (Theorem 1, Sect. 5) has two important implications. First, it shows that the theorem "in the limit" obtained in [6] cannot be explained as the result of a discontinuity. The limit of a sequence of perfect equilibria as frictions converge to zero is Walrasian. Second, indivisibilities are no obstacle to a Walrasian outcome. The model contains the RW model as a special case. Once these two possible explanations are ruled out, we are left with stationarity to account for the RW paradox. In the economy with a finite measure of agents, stationarity is never assumed. In fact, stationarity is generally impossible, since the flow of potential entrants is quite arbitrary but ultimately declines to zero. In the stationary economy, on the other hand, there is assumed to be a constant flow of potential entrants. Since a positive, constant measure of agents enters the market at each date, the set of all agents has infinite measure. The corresponding exchange economy is not well defined. With the infinite measure of buyers and sellers, demand and supply are also infinite. The usual market-clearing condition is mcaningless in this context. Instead, onc has to use some notion of market-clearing on average and this, it turns out, is the key to the RW paradox.

The next step then is to analyse the stationary economy in which the flow of potential entrants is constant. I focus on the stationary perfect equilibria of these models, that is, equilibria in which the distribution of agents by type at each date is constant. This is the exact analogue of the case considered by RW. As before I assume that all agents have the same discount factor and consider the sequence of perfect equilibria corresponding to a sequence of discount factors converging to unity. We are interested in characterizing the limit points. This characterization proceeds in two steps. The first step is to show that in the limit there is a uniform price. That is, every agent exchanges the commodity at the same price, regardless of his type and the date and whether he is proposer or responder. This very strong result by itself shows that the limit point is very close to being a Walrasian equilibrium. What remains to be shown is whether this price is market-clearing. It is worth noting that the uniform price result holds in the original RW model. Because there is only one type of buyer and one type of seller, it is trivially true in a stationary equilibrium that all agents trade at one price.

In stationary states, where the measure of agents passing through the economy is infinite, we are forced to define market-clearing in some average sense. There are two obvious candidates. The first corresponds to a 
concept of stock equilibrium. At each date there is a constant distribution of types of agents in the market. If we abstract this "stock" of agents, we can define a corresponding market-clearing condition in terms of their demands and supplies. According to this condition, a price is market-clearing if it equates the demands and supplies of the buyers and sellers who are in the market at a given date. In Fig. 1, $D$ and $S$ arc the dcmand and supply curves constructed using the data of the agents in the market at a single date. The market-clearing price is $p^{*}$. This is the concept of market-clearing price adopted by RW and others. The alternative notion of market-clearing price corresponds to a concept of flow equilibrium. ${ }^{4}$ Instead of focusing on the stock of agents in the market at a given date we look at the flow of agents into (or out of) the market at that date. A new set of demand and supply curves can be constructed using the data of this set of agents and a new market-clearing price defined by the intersection of the curves. In Fig. 1, these are given by $D^{\prime}$ and $S^{\prime}$ and $p^{* *}$, respectively.

In the market interpretation of the RW model, the market-clearing condition for flow equilibrium is automatically satisfied. By assumption the numbers of buyers and sellers flowing into the market at each date are equal. Since they want to trade one unit each, market-clearing in this sense is satisfied at any price. For this reason flow equilibrium may not seem a very satisfactory concept in the simple case examined by RW. In the more general case represented by Fig. 1, however, flow equilibrium makes a lot of sense. With an arbitrary, constant flow of potential entrants into the market, a stationary equilibrium is possible only if potential entrants can choose whether to enter the market. The price which obtains in the market must be such that the numbers of buyers and sellers choosing to enter are equal. But this condition is met only if the observed price is the flow market-clearing price. As Fig. 1 illustrates, the flow market-clearing condition is both non-trivial and necessary for a stationary equilibrium. It therefore seems the natural concept of market-clearing to use.

There are other considerations which argue for the flow concept as well. Although at any date there is an unsatisfied queue on the long side of the market, every agent does eventually trade. Furthermore, the excess demand or supply, which appears significant at a single date, is constant and therefore negligible relative to the trade carried out over the entire history of the economy.

The most convincing argument against the stock equilibrium concept comes from the formal characterization of the limit point, however. If we take the stock approach first it can be shown that a generalized version of RW's result will hold. For the special case where agents have the same discount factor, the average surplus of agents on either side of the market

\footnotetext{
${ }^{4}$ This concept was introduced in [5].
} 
must be the same in the limit. In terms of Fig. 1, the area below the demand curve $D$ and above the price line $p^{* *}$ is equal to the area above the supply curve $S$ and below the price line $p^{* *}$. If discount factors differed between buyers and sellers, the proportions between these areas would differ accordingly. Note that this result applies to the set of agents actually in the economy at a given date. In general, there is no reason to think that the price $p^{* *}$ which satisfies this relationship will coincide with the stock equilibrium market-clearing price $p^{*}$. So even in this general model there is a variant of the $\mathrm{RW}$ paradox.

At the same time, however, $p^{* *}$ is the market-clearing price in the flow sense. Furthermore it is the flow market-clearing condition which determines $p^{* *}$. Given an arbitrary, constant flow of potential entrants there will generically be a unique price which is consistent with a stationary equilibrium. Thus, in the limit, in the stationary case, the flow of potential entrants uniquely determines the price $p^{* *}$ and hence the payoffs of all the agents. Nothing of consequence is determined by the stock of agents in the market. On the contrary, the stock is determined by the generalized RW condition. In equilibrium, the size of the queue of unsatisfied agents adjusts so that the sum of expected payoffs on both sides of the market is equal. The payoff of each individual agent is unaffected by this condition. This seems to me the strongest possible argument against the use of the stock equilibrium concept. The stock demand and supply curves determine nothing of interest in the generic case and are themselves determined by the flow of potential entrants. This is not to say that the relationship between the stock demand and supply curves and the price is of no interest. In applications, we may be able to observe the stocks but not the potential flows. In that case the Rubinstein-Wolinsky theory comes into its own.

\section{The Bargaining Game}

As I indicated in the Introduction, the economic structure underlying the bargaining game can be taken to represent a market in which an indivisible commodity is traded. On one side of the market we have sellers, each of whom has a single unit of the commodity; on the other side we have buyers, each of whom wants to purchase a single unit of the commodity. Other interpretations are, of course, possible and none of the present interpretation is necessary for the formal analysis or its economic applications.

There is assumed to be a large number (strictly a continuum) of agents. Agents are divided into two classes, buyers and sellers. These classes are then subdivided into a finite number of types of buyers indexed $i=1, \ldots, I$ and a finite number of types of sellers indexed $j=1, \ldots, J$. Where there is no risk of ambiguity, $I$ and $J$ also stand for the sets of types of buyers and 
sellers respectively. Then $H \equiv I \cup J$ denotes the set of all types of agents, both buyers and sellers.

Each buyer is characterized by a limit price. The limit price of the $i$-th type of buyer is denoted by $s_{i} \geqslant 0(i=1, \ldots, I)$. We can think of $s_{i}$ as the utility derived from one unit of the commodity by the ith type. The buyers' types are labelled so that

$$
s_{1}>s_{2}>\cdots>s_{I} .
$$

Each seller is characterized by a reservation price. The reservation price of the $j$ th type of seller is denoted by $t_{j} \geqslant 0(j=1, \ldots, J)$. Think of $j$ as the disutility of parting with one unit of the commodity for the $j$ th type of seller. The sellers' types are labelled so that

$$
t_{1}<t_{2}<\cdots<t_{J} \text {. }
$$

Each seller has a single unit of the good which he wants to sell; each buyer wants to buy precisely one unit. If a seller of the $j$ th type and a buyer of the $i$ th type exchange a single unit of the commodity they create a surplus of $s_{i}-t_{j}$ (which could be negative). All agents are assumed to have transferable utilities so the surplus or gains from trade can be divided in any manner they wish.

An example may help to make the interpretation of these terms absolutely clcar. Suppose the market is a labor market. The buyers are employers; the sellers are workers. Each employer wants to hire at most one worker; each worker wants at most one job. Then $s_{i}$ is the productivity of any worker for the $i$ th type of employer and $t_{i}$ is the disutility of labor (measured in the same units) of the $j$ th type of worker. Other things being equal, employers are indifferent about which type of worker they hire and workers are indifferent about which type of employer hires them.

The play of the game takes place at an infinite sequence of dates. For the moment I shall assume there is an initial date 0 and the set of dates is denoted by $\mathbb{N}=\{0,1,2, \ldots\}$. Later, when it is necessary to consider stationary states, it is convenient to assume there is no first date. I leave the reader to make the appropriate notational adjustments for the second case. Each agent is assigned a single date at which he can enter the market. At that date he can decide either to enter the market or stay out. Because the entry decision is endogenous there exists the possibility of trivial equilibria in which no trade takes place. If every agent decides not to enter the market, no trade is possible. Then it is optimal for every agent to remain outside the market. A similar problem arises if exit is endogenous. If every agent decides to leave the market, then it is optimal for every agent to do so. The exit problem can be resolved, in this model, simply by assuming that, once in the market, agents have to remain in the market until they trade. The entry problem is more intractable. We do not want agents who cannot trade in equilibrium (e.g., because their reservation price is too 
high) to enter the market. The reason is that these agents will accumulate and eventually clog the matching process. Since we cannot prejudge the market's decision about who can and who cannot trade, the entry decision must remain endogenous. So we are stuck with the possibility of trivial equilibria. However, these equilibria are not "stable." If a small measure of agents were accidentally forced into the market, the rest would want to follow, attracted by the gains from trade.

If an agent enters, he must pay a fixed, non-recoverable entry fee $e \geqslant 0$. The entry fee, which is always assumed to be "small," is used to ensure that agents who cannot trade do not enter the market. (cf. Proposition 6 in Sect. 5.) The entry fee is used to avoid the problem of non-trading entrants. (See above.) The fee should be thought of as "small." To obtain the correct equilibrium without a fee we first set $e>0$ and then take limits as $e \rightarrow 0$. Once in the market an agent can remain, searching and bargaining, as long as he likes. Once he has completed a transaction he has to leave and cannot reenter. Before entry and after exit an agent takes no part in the game.

All agents discount future utilities at the same constant rate. Discounting begins at the date when the agent enters the market. For example, an agent who enters the market at date $n$ and completes a transaction at date $N \geqslant n$, receiving a share $z$ of the surplus, has a utility $\alpha^{N-n} \cdot z-e$, where $\alpha$ is the discount factor. It is naturally assumed that

$$
0<\alpha<1 \text {. }
$$

The potential entrants at any date are described by the distribution of their types. Formally, let $P(H)$ denote the power set of $H$ and let $v$ be a function defined on $P(H) \times \mathbb{N}$ to $\mathbb{R}_{+}$. For any set $H^{\prime} \subset H$ and any date $n \in \mathbb{N}$, $v\left(H^{\prime}, n\right)$ is the measure of potential entrants at $n$ whose types belong to $H^{\prime}$. The function $v$ is part of the structure of the model. It is important to remember, however, that $v$ only describes potential entrants at each date. It is up to those agents themselves to decide whether it is worth paying the entry fee $e$ to enter the market.

The structure of the model is defined by the lists of types $I$ and $J$ and the numbers $\left\{s_{i}\right\}_{i \in I}$ and $\left\{t_{j}\right\}_{j \in J}$, by the set of dates $\mathbb{N}$, the discount factor $\alpha$, and by the distribution of potential entrants $v$. The structure $\left\langle I, J,\left\{s_{i}\right\},\left\{t_{j}\right\}, \mathbb{N}, \alpha, v\right\rangle$ satisfying (1) to (3) is denoted by $\mathscr{G}$. The bargaining game is defined by $\mathscr{G}$ together with the specification of the matching process and the bargaining rules. The matching process adopted in this paper is a simple one. It should be clear from the proofs that it is not necessary to restrict the analysis to this particular process. ${ }^{5}$ But there is a

${ }^{5}$ Although the analysis has not been carried out for more general cases it seems likely that what is required is some kind of connectedness property. That is, each type of buyer must have a positive probability of meeting every type of seller that is in the market in significant numbers and vice versa. Of course, for the analysis of steady-states one will have to assume that the matching process is stationary. 
considerable gain in the exposition from choosing such a simple and transparent rule and this, I think, outweighs the apparent lack of generality. The active agents at any date $n$ are those who have already entered the market and have not yet left it, together with the current entrants. For any set $H^{\prime} \subset H$ and any date $n \in \mathbb{N}, \mu\left(H^{\prime}, n\right)$ is the measure of active agents with types in $H^{\prime}$ at date $n$. The function $\mu$ defined on $P(H) \times \mathbb{N}$ to $\mathbb{R}_{+}$is endogenously determined as part of the perfect equilibrium of the bargaining game. It describes completely the population of agents in the market at cach datc. The matching process is a function of $\mu$. Agents are assumcd to be randomly matched in the sense that the probability of meeting an agent of a given type is equal to the proportion of that type in the population. The matching probabilitics are given by a function $p$ defincd on $P(H) \times \mathbb{N}$ to $[0,1]$, where for every $H^{\prime} \subset H$ and $n \in \mathbb{N}$,

$$
p\left(H^{\prime}, n\right)=\mu\left(H^{\prime}, n\right) / \mu(H, n) .
$$

The matching process is serially independent in the sense that an agent's probability of meeting an agent of a given type is independent of what has happened at previous dates. When two agents meet, each has an equal probability of being chosen as a proposer, independently of the matching process.

At any date $n$ an active agent acquires the following information:

(i) he observes whether he has been matched with another agent. If he has been matched, he observes his own type, his partner's type and which of them has been chosen as proposer;

(ii) next he observes the proposer's move, which is a demand for a share $z$ of the surplus generated by trade:

(iii) finally the responder's move is observed (the responder accepts or rejects the proposal).

The assumption that an agent knows his opponent's reservation price or limit price is restrictive. Without this assumption we would have to analyze a bargaining game with incomplete information. The reason for making the assumption of complete information is the following. In the limit, when there is no discounting, there is no scope for inferring an agent's type from his willingness to delay. The only effect of assuming incomplete information is to force an agent to treat all other agents symmetrically. For example, in [7] it is strictly easier to show the bargaining equilibrium is Walrasian under incomplete information than under complete information. So although here we are dealing with a limit theorem rather than a theorem in the limit, it seems that complete information is the more interesting case to study.

An agent's information at a node which he controls consists of everything observed at previous dates since entry, together with what he 
has observed at previous nodes at the current date. In other words, this is a game of perfect recall. A strategy for an agent is a function which prescribes a feasible action at cach nodc he controls. The game is analysed as a subgame perfect Nash equilibrium. Each agent's strategy is a best response to the strategy of other agents at every possible node of the game and not just the nodes observed during the play of the game.

\section{Recursive Equations of the Bargaining Game}

A precise definition of the bargaining game requires a rather weighty formalism which I have here sought to avoid. The analysis of the game, on the other hand, can often be restricted to "soft" or informal arguments. The most important tool in achieving this simplification is the value function which expresses the expected utility of the game to an agent as a function of his type and the current date. The value function satisfies certain recursive equations which bear a strong family resemblance to the functional equations of dynamic programing. These equations can be used to characterize the perfect equilibrium without any direct reference to the potentially complex strategies themselves.

The generic symbol for a value function is $v$. The value function is defined on $H \times \mathbb{N}$ to $\mathbb{R}_{+}$. In this section, I consider a fixed perfect equilibrium of a game with discount factor $\alpha<1$. To emphasize the dependence on $\alpha$, the value function is denoted by $v_{\alpha}$ and the distribution of active agents by $\mu_{x}$. For any $(h, n) \in H \times \mathbb{N}, v_{x}(h, n)$ is the expected utility of an agent of type $h$ at the beginning of date $n$ (i.e., before meeting his bargaining partner for that date). Implicit in the definition of the value function is the fact that an agent's expected utility is independent of his personal history. This fact follows from the assumption that his history is not known to his opponent and that the matching process treats all agents symmetrically. An agent's expected utility is exclusive of the entry fee $e$ and is discounted to the initial date 0 . To obtain the expected utility of an agent who enters the market at some later date $N>0$, simply multiply $v_{\alpha}(h, n)$ by $\alpha^{-N}$.

The functions $v_{x}$ and $\mu_{x}$ contain all the information we need about the pcrfect cquilibrium. It should be clear that if a perfect equilibrium exists, the corresponding functions $v_{\alpha}$ and $\mu_{x}$ are unique and well defined. Recall that $v_{\alpha}$ and $\mu_{\alpha}$ are non-stochastic because there is no aggregate uncertainty. Corresponding to $\left(v_{\alpha}, \mu_{\alpha}\right)$ we have the matching probabilitics $p_{\alpha}$ defined by equation (2.4). ${ }^{6}$ The following proposition establishes the basic recursive equation relating $v_{x}$ and $p_{x}$.

\footnotetext{
${ }^{6}$ In each section equations are numbered $1,2, \ldots$. References to equations in another section are preceded by a section number and a decimal point. Thus equation (4) in Section 2 is referred to as $(2.4)$.
} 
Proposition 1. For any $(i, n) \in I \times \mathbb{N}$,

$$
\begin{aligned}
v_{\alpha}(i, n)= & \left(1-\frac{1}{2} \sum_{j \in J} p_{\alpha}(j, n)\right) v_{x}(i, n+1)+\frac{1}{2} \sum_{j \in J} p_{\alpha}(j, n) \\
& \times \max \left\{\alpha^{n}\left(s_{i}-t_{j}\right)-v_{\alpha}(j, n+1), v_{\alpha}(i, n+1)\right\}
\end{aligned}
$$

and similarly, for any $(j, n) \in J \times \mathbb{N}$,

$$
\begin{aligned}
v_{\alpha}(j, n)= & \left(1-\frac{1}{2} \sum_{i \in I} p_{\alpha}(i, n)\right) v_{\alpha}(j, n+1)+\frac{1}{2} \sum_{i \in I} p_{\alpha}(i, n) \\
& \times \max \left\{\alpha^{n}\left(s_{i}-t_{j}\right)-v_{\alpha}(i, n+1), v_{\alpha}(j, n+1)\right\} .
\end{aligned}
$$

\section{Proof. Omitted.}

The intuition behind the proposition is the following. At any date $n$, one of several things may happen to an agent of the $i$ th typc. He may not trade, in which case he has to wait until the next date and his expected utility is $v_{x}(i, n+1)$. Or he may receive an offer which he accepts. In that case also his expected utility is $v_{x}(i, n+1)$. In a perfect equilibrium he must accept any offer which is better than $v_{x}(i, n+1)$ (his effective security level) so the proposer can reduce his payoff to precisely $v_{\alpha}(i, n+1)$. Finally, the agent may make a proposal which is accepted by an agent of some type $j$. In that case, by the preceding argument, he receives the total surplus $\alpha^{n}\left(s_{i}-t_{j}\right)$ minus what is required to gain the responder's acceptance, namely $v_{x}(j, n+1)$. His expected utility at the beginning of date $n$ is simply the average of his expected utilities in each of these events, weighted by the probabilities of each event. This is precisely what Proposition 1 says.

For the next proposition some additional notation is required. For any function $f$ from $I$ to $\mathbb{R}$ let

$$
A_{i} f(i)=f(i)-f(i+1) .
$$

Thus, for example,

$$
\Delta_{i} s_{i}=s_{i}-s_{i+1}
$$

for $i=1, \ldots, I-1$. The same convention applies to functions defined on $J$, of course.

Proposition 2. For any $(i, n) \in I \times \mathbb{N}$ if $i \leqslant I-1$ then $\Delta_{i} v_{\alpha}(i, n) \leqslant \alpha^{n} \Delta_{i} s_{i}$ and for any $(j, n) \in J \times \mathbb{N}$ if $j \leqslant J-1$ then $\Delta_{j} v_{x}(j, n) \leqslant-\alpha^{n} \Delta_{j} t_{j}$.

Proof. From Eq. (1),

$$
\begin{aligned}
\Delta_{i} v_{\alpha}(i, n)= & \left(1-\frac{1}{2} \sum_{j \in J} p_{\alpha}(j, n)\right) \Delta_{i} v_{\alpha}(i, n+1)+\frac{1}{2} \sum_{j \in J} p_{x}(j, n) \\
& \times \Delta_{i} \max \left\{\alpha^{n}\left(s_{i}-t_{j}\right)-v_{\alpha}(j, n+1), v_{\alpha}(i, n+1)\right\} .
\end{aligned}
$$


For any numbers $a, b, c, d$,

$$
\max \{a, b\}-\max \{c, d\} \leqslant \max \{a-c, b-d\} .
$$

Then, for any $j \in J$,

$$
\begin{aligned}
\Delta_{i} \max & \left\{\alpha^{n}\left(s_{i}-t_{j}\right)-v_{\alpha}(j, n+1), v_{\alpha}(i, n+1)\right\} \\
& \leqslant \max \left\{\Delta_{i}\left(\alpha^{n}\left(s_{i}-t_{j}\right)-v_{x}(j, n+1)\right), \Delta_{i} v_{x}(i, n+1)\right\} \\
& =\max \left\{\alpha^{n} \Delta_{i} s_{i}, \Delta_{i} v_{\alpha}(i, n+1)\right\} .
\end{aligned}
$$

Substitution in Eq. (5) gives

$$
\begin{aligned}
\Delta_{i} v_{\alpha}(i, n) \leqslant & \left(1-\frac{1}{2} \sum_{j \in J} p_{x}(j, n)\right) \Delta_{i} v_{\alpha}(i, n+1)+\frac{1}{2} \sum_{j \in J} p_{x}(j, n) \\
& \times \max \left\{\alpha^{n} \Delta_{i} s_{i}, \Delta_{i} v_{\alpha}(i, n+1)\right\} .
\end{aligned}
$$

Now suppose, contrary to what we want to prove, that $\Delta_{i} v_{x}(i, n)>\alpha^{n} \Delta_{i} s_{i}$. Inequality (6) implies that $\Delta_{i} v_{x}(i, n+1) \geqslant \Delta_{i} v_{x}(i, n)$. Otherwise the inequality could only be satisfied if $A_{i} v_{x}(i, n+1)<\alpha^{n} \Delta_{i} s_{i}$, in which case, since $0 \leqslant \frac{1}{2} \sum_{j \in J} p_{x}(j, n) \leqslant 1$, the inequality would imply $\Delta_{i} v_{\alpha}(i, n) \leqslant \alpha^{n} \Delta_{i} s_{i}$, a contradiction. Because (6) holds for all $n$, it follows by induction on $n$ that $v_{\alpha}(i, n+k) \geqslant v_{x}(i, n+k-1)$ for all $k \geqslant 1$. Then

$$
\lim _{k \rightarrow \infty} \Delta_{i} v_{\alpha}(i, n+k) \geqslant \alpha^{n} \Delta_{i} s_{i}>0 .
$$

However, the fact that $v_{x}(h, n) \geqslant 0$ for all $(h, n) \in H \times \mathbb{N}$ and the surplus to be divided converges to zero as $n \rightarrow \infty$ when $\alpha<1$ implies that $\lim _{k \rightarrow \infty} v_{x}(i, n+k)=0$, a contradiction. Thus, $\Delta_{i} v_{x}(i, n) \leqslant \alpha^{n} \Delta_{i} s_{i}$. The proof that $\Delta_{j} v_{x}(j, n) \leqslant-\alpha^{n} \Delta_{j} t_{j}$ is similar.

Proposition 2 shows that an agent cannot obtain more from the bargaining game than he contributes. In a competitive equilibrium he gets exactly what he contributes. When $\alpha<1$, on the other hand, he may get less because he happens to be the responder and can do no better than if agreement had not been reached.

\section{Limit Points of Perfect Equilibria}

All the information we need about a perfect equilibrium is contained in the value function $v$ and the distribution $\mu$. Let $A=\left\{\alpha_{1}, \alpha_{2}, \ldots\right\}$ be a sequence of numbers in $(0,1)$ converging to 1 . For any $\alpha \in A$, let $\left(v_{\alpha}, \mu_{\alpha}\right)$ denote the values of $v$ and $\mu$ corresponding to some perfect equilibrium of the game (assuming one exists) with discount factor $\alpha$. The sequence 
$\left\{\left(v_{\alpha}, \mu_{\alpha}\right)\right\}_{\alpha \in A}$ has a pointwise convergent subsequence which can be taken to be the original sequence. Let

$$
(v, \mu)=\lim _{k \rightarrow \infty}\left(v_{x_{k}}, \mu_{x_{k}}\right) .
$$

Note that $(v, \mu)$ is not necessarily an equilibrium of the game with $\alpha=1$; $(v, \mu)$ simply represents the limiting behavior of a sequence of equilibria as frictions vanish. For any $H^{\prime} \subset H$ and $n \in \mathbb{N}$ define

$$
p\left(H^{\prime}, n\right)=\mu\left(H^{\prime}, n\right) / \mu(H, n) .
$$

From (2.4) and the convergence of $\left\{\mu_{\alpha}\right\}$ it follows that $p_{\alpha}\left(H^{\prime}, n\right) \rightarrow p\left(H^{\prime}, n\right)$ as $\alpha \rightarrow 1$ for any $H^{\prime} \subset H$ and $n \in \mathbb{N}$.

With these definitions one can immediately state analogues "in the limit" of the results derived in Section 3. In each case the result follows from the continuity of the relationship and the convergence of $\left\{\left(v_{x}, \mu_{x}\right)\right\}_{x \in A}$. From Proposition 1 we have, for any $(i, n) \in I \times \mathbb{N}$,

$$
\begin{aligned}
v(i, n)= & \left(1-\frac{1}{2} \sum_{j \in J} p(j, n)\right) v(i, n+1)+\frac{1}{2} \sum_{j \in J} p(j, n) \\
& \times \max \left\{s_{i}-t,-v(j, n+1), v(i, n+1)\right\}
\end{aligned}
$$

and for any $(j, n) \in J \times \mathbb{N}$,

$$
\begin{aligned}
v(j, n)= & \left(1-\frac{1}{2} \sum_{i \in I} p(i, n)\right) v(j, n+1)+\frac{1}{2} \sum_{i \in I} p(i, n) \\
& \times \max \left\{s_{i}-t_{j}-v(i, n+1), v(j, n+1)\right\} .
\end{aligned}
$$

It is immediate from (3) and (4) that $v(h, n) \geqslant v(h+1, n) \geqslant 0$ for any $(h, n) \in H \times \mathbb{N}$. Let $v(h, \infty)$ denote the limit as $n \rightarrow \infty$ of $v(h, n)$ for any $h \in H$.

Proposition 3. For any $(i, j) \in I \times J$ if either $\lim _{n} \sup p(i, n)>0$ or $\lim _{n} \sup p(j, n)>0$ then $\left(s_{i}-t_{j}\right) \leqslant v(i, \infty)+v(j, \infty)$.

Proof. Suppose that $\lim _{n} \sup p\left(i_{0}, n\right)>0$ and let $p(\cdot, \infty)$ denote a limit point of $\{p(\cdot, n)\}_{n \in \mathbb{N}}$ such that $p\left(i_{0}, \infty\right)>0$. From (4) and continuity,

$$
\begin{aligned}
v(j, \infty)= & \left(1-\frac{1}{2} \sum_{i \in I} p(i, \infty)\right) v(j, \infty)+\frac{1}{2} \sum_{i \in I} p(i, \infty) \\
& \times \max \left\{s_{i}-t_{j}-v(j, \infty), v(i, \infty)\right\},
\end{aligned}
$$

which implies that

$$
0=\sum_{i \in I} p(i, \infty) \max \left\{s_{i}-t_{j}-v(i, \infty)-v(j, \infty), 0\right\}
$$


Since $p\left(i_{0}, \infty\right)>0, s_{i_{0}}-t_{j}-v\left(i_{0}, \infty\right)-v(j, \infty) \leqslant 0$ as required. The other case is handled similarly.

Corollary. For any $h \in H$ if $\lim _{n} \sup p(h, n)>0$ then $v(h, n)=$ $v(h, n+1)$ for any $n \in \mathbb{N}$.

Proof. Suppose that $\lim _{n} \sup p(i, n)>0$ for some $i \in I$. Then from Proposition $3, s_{i}-t_{j} \leqslant v(i, \infty)+v(j, \infty)$ for any $j \in J$. Since $v(h, n)$ is monotonically non-increasing in $n$ for any $h \in H, s_{i}-t_{j} \leqslant v(i, n)+v(j, n)$ for any $n \in \mathbb{N}$ and for any $j \in J$. From inspection of (3) it appears that $v(i, n)=v(i, n+1)$ for all $n \in \mathbb{N}$. The other case is similar.

From Proposition 2 we have, for any $(i, n) \in I \times \mathbb{N}$,

$$
\Delta_{i} v(i, n) \leqslant \Delta_{i} s_{i}
$$

and for any $(j, n) \in J \times \mathbb{N}$,

$$
\Delta_{j} v(j, n) \leqslant-\Delta_{j} t_{j} .
$$

In competitive equilibrium these inequalities hold as strict equalities for agents who trade. As a first step toward establishing this result, I derive a lower bound for $A_{h} v(h, n)$ in the next two propositions. First, some more notation is needed. For any $(i, j, n) \in I \times J \times \mathbb{N}$ define

$$
\psi(i, j, n)= \begin{cases}p(j, n) & \text { if } s_{i}-t_{j} \geqslant v(i, n+1)+v(j, n+1) \\ 0 & \text { otherwise }\end{cases}
$$

and

$$
\psi(j, i, n)= \begin{cases}p(i, n) & \text { if } s_{i}-t_{j} \geqslant v(i, n+1)+v(j, n+1) \\ 0 & \text { otherwise. }\end{cases}
$$

For any $(i, n) \in I \times \mathbb{N}$ define

$$
\phi(i, n)=\prod_{k=0}^{n-1}\left(1-\frac{1}{2} \sum_{j \in J} \psi(i, j, k)\right)
$$

and for any $(j, n) \in J \times \mathbb{N}$ define

$$
\phi(j, n)=\prod_{k=0}^{n-1}\left(1-\frac{1}{2} \sum_{i \in I} \psi(j, i, k)\right) .
$$

For any $(i, j, n) \in I \times J \times \mathbb{N}, \psi(i, j, n)$ is the probability that an agent of type $i$, who is in the market at date $n$, meets an agent of type $j$ and can trade with him. The interpretation of $\psi(j, i, n)$ is similar. For any $(i, n) \in I \times \mathbb{N}$, $\phi(i, n)$ is the probability that an agent of type $i$, who is in the market at 
date 0 , will not propose to anyone with whom he can trade before date $n$. The interpretation of $\phi(j, n)$ is similar.

Proposition 4. For any $(i, n) \in I \times \mathbb{N}$,

$$
\begin{aligned}
v(i, n)= & \phi(i, n)^{-1} \sum_{k=n}^{N} \phi(i, k) \frac{1}{2} \sum_{j \in J} \psi(i, j, k)\left(s_{i}-t_{j}-v(j, k+1)\right) \\
& +\phi(i, n)^{-1} \phi(i, N+1) v(i, N+1)
\end{aligned}
$$

and, similarly, for any $(j, n) \in J \times \mathbb{N}$,

$$
\begin{aligned}
v(j, n)= & \phi(j, n)^{-1} \sum_{k=n}^{N} \phi(j, k) \frac{1}{2} \sum_{i \in I} \psi(j, i, k)\left(s_{i}-l_{j}-v(i, k+1)\right) \\
& +\phi(j, n)^{-1} \phi(j, N+1) v(j, N+1) .
\end{aligned}
$$

Proof. The proof is by induction on $N$. For any $(i, n) \in I \times \mathbb{N}$, the expansion for $v(i, n)$ is identical with Eq. (3) when $N=n$. Now suppose it is valid for some $N \geqslant n$. Then

$$
\begin{aligned}
v(i, n)= & \phi(i, n)^{-1} \sum_{k=n}^{N} \phi(i, k) \frac{1}{2} \sum_{j \in J} \psi(i, j, k)\left(s_{i}-t_{j}-v(i, k+1)\right) \\
& +\phi(i, n)^{-1} \phi(i, N+1) v(i, N+1) \\
= & \phi(i, n)^{-1} \sum_{k=n}^{N} \phi(i, k) \frac{1}{2} \sum_{j \in J} \psi(i, j, k)\left(s_{i}-t_{j}-v(i, k+1)\right) \\
& +\phi(i, n)^{-1} \phi(i, N+1)\left\{\frac{1}{2} \sum_{j \in J} \psi(i, j, N+1)\left(s_{i}-t_{j}-v(j, N+2)\right)\right. \\
& \left.+\left(1-\frac{1}{2} \sum_{j \in J} \psi(i, j, N+1)\right) v(i, N+2)\right\} \\
= & \phi(i, n)^{-1} \sum_{k=n}^{N+1} \phi(i, k) \frac{1}{2} \sum_{j \in J} \psi(i, j, k)\left(s_{i}-t_{j}-v(i, k+1)\right) \\
& +\phi(i, n)^{-1} \phi(i, N+2) v(i, N+2) .
\end{aligned}
$$

The expansion for $v(i, n)$ is thus valid for all $N \geqslant n$. The proof for $v(j, n)$ is similar.

Proposition 5. For any $i=1, \ldots, I-1$ and $n \in \mathbb{N}$,

$$
\begin{aligned}
\Delta_{i} v(i, n) \geqslant & \phi(i+1, n)^{-1} \sum_{k=n}^{N} \phi(i+1, k) \frac{1}{2} \sum_{j \in J} \psi(i, j, k) \Delta_{i} s_{i} \\
& +\phi(i+1, n)^{-1} \phi(i+1, N+1) \Delta_{i} v(i, N+1) .
\end{aligned}
$$


Similarly, for any $j=1, \ldots, J-1$ and $n \in \mathbb{N}$,

$$
\begin{aligned}
\Delta_{j} v(j, n) \geqslant & \phi(j+1, n)^{-1} \sum_{k=n}^{N} \phi(j+1, k) \frac{1}{2} \sum_{j \in J} \psi(i, j, k)\left(-\Delta_{j} t_{j}\right) \\
& +\phi(j+1, n)^{-1} \phi(j+1, N+1) \Delta_{j} v(j, N+1) .
\end{aligned}
$$

Proof. Once again the proof is by induction and I only prove the first inequality. The inequality is an identity for any $i=1, \ldots, I-1, n \in \mathbb{N}$ and $N=n-1$. Suppose it is true for some $N \geqslant n-1$. Then

$$
\begin{aligned}
\Delta_{i} v(i, n) \geqslant & \phi(i+1, n)^{-1} \sum_{k=n}^{N} \phi(i+1, k) \frac{1}{2} \sum_{j \in J} \psi(i, j, k) \Delta_{j} s_{j} \\
& +\phi(i+1, n)^{-1} \phi(i+1, N+1) \Delta_{i} v(i, N+1) \\
= & \phi(i, n)^{-1} \sum_{k=n}^{N} \phi(i+1, k) \frac{1}{2} \sum_{j \in J} \psi(i, j, k) \Delta_{i} s_{i} \\
& +\phi(i+1, n)^{-1} \phi(i+1, N+1) \\
& \times \Delta_{i}\left\{\left(1-\frac{1}{2} \sum_{j \in J} \psi(i, j, N+1)\right) v(i, N+2)\right. \\
& \left.+\frac{1}{2} \sum_{j \in J} \psi(i, j, N+1)\left(s_{i}-t_{j}-v(j, N+2)\right)\right\} .
\end{aligned}
$$

Now

$$
\begin{aligned}
\Delta_{i}\{(1 & \left.-\frac{1}{2} \sum_{j \in J} \psi(i, j, N+1)\right) v(i, N+2) \\
& \left.+\frac{1}{2} \sum_{j \in J} \psi(i, j, N+1)\left(s_{i}-t_{j}-v(j, N+1)\right)\right\} \\
= & \left(1-\frac{1}{2} \sum_{j \in J} \psi(i+1, j, N+1)\right) \Delta_{i} v(i, N+2) \\
& -\frac{1}{2} \sum_{j \in J} \Delta_{i} \psi(i, j, N+1) v(i, N+2)+\frac{1}{2} \sum_{j \in J} \psi(i+1, j, N+1) \Delta_{i} s_{i} \\
& +\frac{1}{2} \sum_{j \in J} \Delta_{i} \psi(i, j, N+1)\left(s_{i}-t_{j}-v(j, N+1)\right) .
\end{aligned}
$$

From (5) and the definition of $\psi$ it follows that $\Delta_{i} \psi(i, j, N+1) \geqslant 0$ and that $A_{i} \psi(i, j, N+1)>0$ implies $s_{i}-t_{j}-v(i, N+1)-v(j, N+1) \geqslant 0$. Thus,

$$
\frac{1}{2} \sum_{j \in J} \Delta_{i} \psi(i, j, N+1)\left(s_{i}-t_{j}-v(i, N+1)-v(j, N+1)\right) \geqslant 0
$$


and (8) reduces to

$$
\begin{aligned}
\Delta_{i}\{(1 & \left.-\frac{1}{2} \sum_{j \in J} \psi(i, j, N+1)\right) v(i, N+2) \\
& \left.+\frac{1}{2} \sum_{j \in J} \psi(i, j, N+1)\left(s_{i}-t_{j}-v(j, N+1)\right)\right\} \\
\geqslant & \left(1-\frac{1}{2} \sum_{j \in J} \psi(i+1, j, N+1)\right) \Delta_{i} v(i, N+2) \\
& +\frac{1}{2} \sum_{j \in J} \psi(i+1, j, N+1) \Delta_{i} s_{i} .
\end{aligned}
$$

Substituting this inequality into (7) produces the desired result for $N+1$ and, by induction, for all $N+1$.

\section{Economies with Finite Measure}

In this section the results obtained in Sections 3 and 4 are applied to the case of an economy in which the total measure of all potential entrants is finite. This case is important for several reasons. First, with a finite measure of agents the economy has a well-defined set of competitive equilibria. In the limit, as $\alpha \rightarrow 1$, the perfect equilibria of the bargaining game implement one of these equilibria. When the measure of agents is infinite there is some ambiguity about the definition of competitive equilibrium and the available limit theorems are therefore harder to interpret. Second, the finite measure case is a particularly tractable example in which the proportions of agents of different types change over time as a result of trade. These endogenous changes in the distribution of agents are an important factor in ensuring the competitive outcome. Third, the assumption of a finite measure of agents was used in [7] to obtain a theorem "in the limit" for a bargaining game based on a very general exchange economy. The "limit theorem" obtained in this section can be seen as complementary to the result obtained in [7], at least for this very special class of games. It shows that the limit economy studied in [7] is a good approximation to economies with small but positive discount rates and a finite measure of agents. Of course, one could also prove a theorem "in the limit" for the economy described in Section 4, i.e., prove that Theorem 1 is true for $\alpha=1$.

The crucial assumption used in this section is that

$$
v(H, n)=0 \quad \text { for } \quad n=1,2, \ldots
$$

(The main theorem of this section is also true under the assumption that 
$\sum_{n \in \mathbb{N}} v(H, n)<\infty$. That case corresponds more exactly to the entry assumptions made in [6] but otherwise does not add extra insight. The more limited assumption (1) is made to simplify the analysis.) From the definition of $\mu_{x}$ it is clear that for any $H^{\prime} \subset H$ and $n \in \mathbb{N}, \mu_{x}\left(H^{\prime}, n\right) \geqslant$ $\mu_{x}\left(H^{\prime}, n+1\right)$. Taking limits as $\alpha \rightarrow 1$ gives

$$
\mu\left(H^{\prime}, n\right) \geqslant \mu\left(H^{\prime}, n+1\right)
$$

for any $H^{\prime} \subset H$ and $n \in \mathbb{N}$. It follows immediately that, for any $H^{\prime} \subset H$,

$$
\lim _{n \in \mathbb{N}} \mu\left(H^{\prime}, n\right)=\mu\left(H^{\prime}, \infty\right)
$$

exists. Equation (3) immediately implies via (4.4) that for any $H^{\prime} \subset H$,

$$
\lim _{n \in \mathbb{N}} p\left(H^{\prime}, n\right)=p\left(H^{\prime}, \infty\right)
$$

exists.

The value of $p(\cdot, \infty)$ has important implications for the value function $v$, as we saw in Section 4 . The sequence of propositions below considers different possibilities concerning $\mu(\cdot, \infty)$ and $p(\cdot, \infty)$ in order to characterize $v$ completely.

Proposition 6. Suppose that $e>0$ and the economy has finite measure. For any $h \in H$ such that $\mu(h, \infty)>0, \phi(h, n) \rightarrow 0$ as $n \rightarrow \infty$.

Proof. The sum of the expected utilities of the active agents in the market at any date cannot exceed the total surplus that could be produced if those agents were immediately matched in an optimal way. Thus, for any $\alpha$ and $n \in \mathbb{N}$ there must exist numbers $x_{\alpha}(i, j, n) \geqslant 0$ for every $(i, j) \in I \times J$ such that

$$
\sum_{h \in H} \mu_{\alpha}(h, n) v_{\alpha}(h, n) \leqslant \sum_{i \in I} \sum_{j \in J} x_{\alpha}(i, j, n)\left(s_{i}-t_{j}\right)
$$

and such that

$$
\sum_{j \in J} x_{\alpha}(i, j, n) \leqslant \mu_{\alpha}(i, n) \quad \text { for each } i \in I
$$

and

$$
\sum_{i \in I} x_{x}(i, j, n) \leqslant \mu_{x}(j, n) \quad \text { for each } \quad j \in J
$$


Taking limits, first as $\alpha \rightarrow 1$ and then as $n \rightarrow \infty$, we see that there must exist a number $x(i, j) \geqslant 0$ for every $(i, j) \in I \times J$ such that

$$
\sum_{h \in H} \mu(h, \infty) v(h, \infty) \leqslant \sum_{i \in l} \sum_{j \in J} x(i, j)\left(s_{i}-t_{j}\right)
$$

where

$$
\sum_{j \in J} x(i, j) \leqslant \mu(i, \infty) \quad \text { for each } \quad i \in I
$$

and

$$
\sum_{i \in I} x(i, j) \leqslant \mu(j, \infty) \quad \text { for each } j \in J .
$$

From (3) it follows that $\mu(I, \infty)>0$ if and only if $\mu(J, \infty)>0$. To see this suppose that $\mu(I, \infty)>0$ and $\mu(J, \infty)=0$. (The case where $\mu(J, \infty)>0$ and $\mu(i, \infty)=0$ is exactly similar.) Then (3) implies that $v(i, \infty)=0$ for any $i \in I$ such that $\mu(i, \infty)>0$ and, by the corollary to Proposition $3, v(i, n)=0$ for all $n \in \mathbb{N}$. But this means that for all $\alpha$ sufficiently large $v_{\alpha}(i, 0)<e$. No agent of type $i$ will enter the market, $\mu_{x}(i, n)=0$ for all $n \in \mathbb{N}$ and $\alpha$ sufficiently large and so $\mu(i, n)=0$ for all $n \in \mathbb{N}$, a contradiction.

From Proposition 3, if $\mu(i, \infty)>0$ or $\mu(j, \infty)>0$ then $s_{i}-t_{j}-v(i, \infty)-$ $v(j, \infty) \leqslant 0$. But this together with (3) implies that for any $(i, j) \in I \times J$, if $\mu(i, \infty)>0$ and $\mu(j, \infty)>0$ then $s_{i}-t_{j}-v(i, \infty)-v(j, \infty)=0$. By the corollary to Proposition 3, for any $n \in \mathbb{N}, s_{i}-t_{j}-v(i, n)-v(j, n)=0$. Then, for any $i \in I$ such that $\mu(i, \infty)>0$,

$$
\limsup _{n} \sum_{j \in J} \psi(i, j, n)>0,
$$

which implies that $\lim _{n \rightarrow \infty} \phi(i, n)=0$. The analogous result obviously holds for any $j \in J$ such that $\mu(j, \infty)>0$.

Proposition 7. For any $h \in H, \mu(h, n)>0$ and $\mu(h, \infty)=0$ implies that $\phi(h, \infty)=0$.

Proof. For any $\alpha \in A$ and $n \in \mathbb{N}$, the probability that an agent of type $i \in I$, who is active at date $n$, leaves the market at date $n$ is less than or equal to $\sum_{j \in J} \psi_{\alpha}(i, j, n)$, the probability of meeting an agent with whom trade is possible. Then

$$
\mu_{\alpha}(i, n+1) \geqslant\left(1-\sum_{i \in J} \psi_{\alpha}(i, j, n)\right) \mu_{\alpha}(i, n)
$$


since the right-hand side is a lower bound for the measure of agents of type $i$ remaining in the market from date $n$ to date $n+1$.

Taking limits as $\alpha \rightarrow 1$,

$$
\mu(i, n+1) \geqslant\left(1-\sum_{j \in J} \psi(i, j, n)\right) \mu(i, n)
$$

and, immediately,

$$
\mu(i, n) \geqslant \prod_{k=0}^{n-1}\left(1-\sum_{j \in J} \psi(i, j, k)\right) \mu(i, 0) .
$$

Suppose that for some $i \in I, \mu(i, 0)>0$ and $\mu(i, \infty)=0$. (The general case $\mu(i, n)>0$ is treated in the same way.) Then from (4) it follows that

$$
\prod_{k=0}^{\infty}\left(1-\sum_{j \in J} \psi(i, j, k)\right)=0 \text {. }
$$

For every value of $\left.k, \sum_{j \in J} \psi(i, j, k)\right)<1$. This is certainly true for $k=0$ since $\mu(i, 0)>0$ by hypothesis. If we assume that $\Sigma_{j \in J} \psi(i, j, k)<1$ for $k=$ $0, \ldots, n-1$ then (4) implies $\mu(i, n)>0$ so $\sum_{j \in J} \psi(i, j, n)<1$. Thus, the claim follows by induction. Given that the terms in the product in (5) are all positive, a necessary and sufficient condition for $(5)$ is that

$$
\sum_{k=0}^{\infty} \sum_{j \in J} \psi(i, j, k)=\infty
$$

in which case it follows by the same criterion that

$$
\prod_{k=0}^{\infty}\left(1-\frac{1}{2} \sum_{j \in J} \psi(i, j, k)\right)=0
$$

as required. The proof for $j \in J$ is exactly similar.

Propositions 6 and 7 show that for any agent who is active in the market at some date, $\phi(h, n) \rightarrow 0$ as $n \rightarrow \infty$. From Proposition 5 it follows that for any $i \in I$ such that $i+1$ enters the market,

$$
\Delta_{i} v(i, n) \geqslant \phi(i+1, n)^{-1} \sum_{k=n}^{\infty} \phi(i+1, k) \frac{1}{2} \sum_{j \in J} \psi(i, j, k) \Delta_{i} s_{i}
$$

and similarly, for any $j \in J$ such that $j+1$ enters the market,

$$
\Delta_{j} v(j, n) \geqslant \phi(j+1, n)^{-1} \sum_{k=n}^{\infty} \phi(j+1, k) \frac{1}{2} \sum_{i \in I} \psi(j, i, k)\left(-\Delta_{j} \iota_{j}\right) .
$$


From the definition of $\phi$ however it follows by direct calculation that for any $i \in I$ such that $i+1$ enters the market,

$$
\phi(i, n)^{-1} \sum_{k=n}^{\infty} \phi(i, k) \frac{1}{2} \sum_{i \in J} \psi(i, j, k)=1-\phi(i, \infty)=1,
$$

and similarly for $j \in J$, if $j+1$ enters the market. Hence, for any $i \in I$ such that $i+1$ enters

$$
\Delta_{i} v(i, n) \geqslant \Delta_{i} s_{i}
$$

and for any $j \in J$ such that $j+1$ enters

$$
\Delta_{j} v(j, n) \geqslant-\Delta_{j} t_{j}
$$

The inequalities (8) and (9) taken in conjunction with (4.5) and (4.6) imply that for any $i \in I$ (resp. $j \in J$ ) such that $i+1$ (resp. $j+1$ ) is in the market, $\Delta_{i} v(i, n)=\Delta_{i} s_{i}\left(\right.$ resp. $\left.\Delta_{j} v(j, n)=-\Delta_{j} t_{j}\right)$.

Proposition 8. If $e>0$ and the economy has finite measure there exists $\omega \geqslant 0$ such that, for any $i \in I$ satisfying $\mu(i, n)>0$ for some $n \in \mathbb{N}$,

$$
v(i, n)=s_{i}-\omega \quad \text { for all } n \in \mathbb{N},
$$

and, for any $j \in J$ satisfying $\mu(j, n)>0$ for some $n \in \mathbb{N}$,

$$
v(j, n)=\omega-t_{j} \quad \text { for all } n \in \mathbb{N} .
$$

Proof. Let $i_{0}$ be the largest index $i \in I$ satisfying $\mu(i, n)>0$ for some $n \in \mathbb{N}$. Define $j_{0}$ similarly. I claim that, for any $n \in \mathbb{N}$,

$$
v\left(i_{0}, n\right)+v\left(j_{0}, n\right)=s_{i_{0}}-t_{j 0} .
$$

To see this, suppose first that, for some $n \in \mathbb{N}$,

$$
v\left(i_{0}, n\right)+v\left(j_{0}, n\right)>s_{i_{0}}-t_{j 0} .
$$

Then for any $i \leqslant i_{0}$ and $j \leqslant j_{0}$,

$$
v(i, n)+v(j, n)>s_{i}-t_{j}
$$

since $\Delta_{i} v(i, n)=\Delta_{i} s_{i}$ for $i<i_{0}$ and $\Delta_{j} v(j, n)=-\Delta_{j} t_{j}$ for $j<j_{0}$. But since $\Delta_{i} v(i, n) \leqslant \Delta_{i} s_{i}$ and $\Delta_{j} v(j, n) \leqslant-\Delta_{j} t_{j}$ for all $i \in I$ and $j \in J$ it follows that (10) holds for all $i \in I$ and $j \in J$. Consequently, no trade is possible. From the fundamental recursive equations (4.3) and (4.4) it is clear that (10) holds for all $n^{\prime} \geqslant n$. But this implies that $v\left(i_{0}, n\right)=0$ and $v\left(j_{0}, n\right)=0$, contradicting $e>0$. 
Now consider the other case, where

$$
v\left(i_{0}, n\right)+v\left(j_{0}, n\right)<s_{i_{0}}-t_{j 0} .
$$

Then, for any $i \leqslant i_{0}$ and $j \leqslant j_{0}$,

$$
v(i, n)+v(j, n)<s_{i}-t_{j}
$$

Then for any triple $(i, j, k) \in I \times J \times \mathbb{N}$ we know that $s_{i}-t_{j}-v(i, k+1)$ is independent of $j$, for any $(i, j, k)$ such that $\psi(i, j, k)>0$, and non-increasing in $k$. Then Proposition 4 indicates that $v(i, n)$ is non-decreasing over time, contradicting (11) and (4.3).

The proposition follows directly from $v\left(i_{0}, n\right)+v\left(j_{0}, n\right)=s_{i_{0}}-t_{i_{0}}$ and $\Delta_{i} v(i, n)=\Delta_{i} s_{i}$ for $i<i_{0}$ and $\Delta_{j} v(j, n)=-\Delta_{j} t_{j}$ for $j<j_{0}$.

To complete the characterization of perfect equilibrium it remains to show that $\omega$ is a market-clearing price. A potential entrant of type $h$ at date $n$ will enter if $v(h, n)>e$ and stay out if $v(h, n)<e$. He is indifferent if $v(h, n)=e$. Demand and supply are equal at the distribution $v$ if and only if the following conditions are satisfied. Let

$$
i_{0}=\max \{i \in I \mid v(i, n) \geqslant e\} \quad \text { and } \quad j_{0}=\max \{j \in J \mid v(j, n) \geqslant e\} .
$$

Then either

with

$$
\sum_{n \in \mathbb{N}} \sum_{i=1}^{i_{0}-1} v(i, n) \leqslant \sum_{n \in \mathbb{N}} \sum_{j=1}^{j_{0}} v(j, n) \leqslant \sum_{n \in \mathbb{N}} \sum_{i=1}^{i_{0}} v(i, n)
$$

$$
v\left(i_{0}, n\right)=e \quad \text { if the last inequality is strict }
$$

or else

$$
\sum_{n \in \mathbb{N}} \sum_{j=1}^{j_{0}-1} v(j, n) \leqslant \sum_{n \in \mathbb{N}} \sum_{i=1}^{i_{0}} v(i, n) \leqslant \sum_{n \in \mathbb{N}} \sum_{j=1}^{j_{0}} v(j, n)
$$

with

$$
v\left(j_{0}, n\right)=e \quad \text { if the last inequality is strict. }
$$

To see that either (12) or (13) must be satisfied suppose the contrary. There are several possible cases to be considered. It may be that one of the inequalities in both (12) and (13) is violated. In that case either

$$
\sum_{n \in N} \sum_{i=1}^{i_{0}} v(i, n)<\sum_{n \in N} \sum_{j=1}^{j_{0}-1} v(j, n)
$$

or

$$
\sum_{n \in N} \sum_{j=1}^{j_{0}} v(j, n)<\sum_{n \in N}^{N} \sum_{i=1}^{i_{0}-1} v(i, n)
$$


Suppose (14) holds. For some small $\varepsilon>0$ and some large $N \in \mathbb{N}$,

$$
\sum_{n=0}^{N} \sum_{i=1} v(i, n)+2 \varepsilon<\sum_{n=0}^{N} \sum_{j=1}^{j 0} v(j, n)
$$

and

$$
\sum_{n=N+1}^{\infty} \sum_{i \in I} v(i, n)<\varepsilon
$$

For $n \leqslant N$ and all $\alpha$ sufficiently large, $v_{\alpha}(i, n)<e$ for $i>i_{0}$ and $v_{\alpha}(j, n)>e$ for $j<j_{0}$. Consequently, for all $\alpha$ sufficiently large there must be a surplus of sellers of measure $2 \varepsilon$ or more at date $N$. Since the measure of buyers entering after $N$ is bounded by $\varepsilon$ we have $\phi_{\alpha}(j, N) \geqslant \frac{1}{2}$ for some $j<j_{0}$ and $\alpha$ sufficiently large. In the limit as $\alpha \rightarrow 1, \phi(j, N) \geqslant \frac{1}{2}$ for some $j<j_{0}$. Since $N$ is arbitrary, this contradicts Proposition 7. By an exactly similar argument (15) leads to contradiction. This shows that the inequalities in either (12) or (13) must be satisfied. If neither (12) nor (13) holds it must be because the complementary slackness conditions are not satified. Suppose then that the inequalities in (12) hold, the second one strictly, but $v\left(i_{0}, n\right)>e$. By the preceding argument one again shows there is a permanent surplus of buyers of types $i \leqslant i_{0}$ which contradicts Proposition 7. (13) is established the same way.

The following theorem has been proved.

THEOREM 1. Let $\left\{\left(v_{\alpha}, \mu_{\alpha}\right)\right\}$ be a sequence of perfect equilibria for an economy with a finite measure of agents and a positive entry fee. If $(v, \mu)$ is a limit point of the sequence then $(v, \mu)$ is Walrasian in the sense that, for some price $\omega$,

$$
\begin{aligned}
& v(i, n)>e \Rightarrow v(i, n)=s_{i}-\omega \\
& v(j, n)>e \Rightarrow v(j, n)=\omega-t_{j}
\end{aligned}
$$

and the market-clearing conditions (12) and (13) are satisfied.

\section{Economies With Infinite Measure: Steady States}

The analysis of economies with finite measure can be treated as a nontatonnement theory of how an economy reaches the Walrasian equilibrium. This kind of theory is useful for some purposes but it is not well suited to describing a market which operates in real time. An obvious feature of the economy with a finite measure of agents is that the population is cventually depleted. In actual markets the number of active participants may fluctuate 
but there is no long-run tendency for the population to disappear. On average, departing agents are balanced by newcomers. Incorporating this fact into the present framework means that the measure of all the agents who are active in the economy at some date is infinite. To analyse this case in the most general terms is difficult. Because the measure of buyers and sellers, summed over all dates, is infinite, attainability conditions are meaningless. It is the absence of these conditions, which were used extensively in the finite case, that explains the greater difficulty of analysing the economy with infinite measure. In special cases, however, the analysis is quite tractable. If we restrict the analysis to steady states it is easy to characterize the limit points of sequences of perfect equilibria.

Two kinds of steady state analysis are possible, differing only in the assumptions made about entry. The first kind makes use of an assumption introduced by Rubinstein-Wolinsky [13]. At the first date $(n=0)$ there is an exogenously determined distribution of types denoted by $\mu_{0}$. These agents are all active (there is no cost to entering the market). At each subsequent date agents leave the market in pairs, one buyer for every seller. The crucial assumption is that every agent who leaves the market is immediately replaced by an agent of the same type. Thus, at each date $n \in \mathbb{N}$ the distribution of active agents is

$$
\mu(\cdot, n)=\mu_{0} .
$$

In this case it is convenient to assume there is no entry fee: $e=0$.

Let $(v, \mu)$ be a limit point of a sequence $\left\{v_{\alpha}, \mu_{\alpha}\right\}_{\alpha \in A}$ of perfect equilibria as described in Section 4. If (1) is satified for every $\alpha \in A$ it will obviously be satisfied in the limit. The results obtained in Section 4 clearly apply to this case too. From the Corollary to Proposition 3 and the definition of the matching probabilities, it is immediate that the value function is timeinvariant. To be more precise, for any $h \in H$ such that $\mu_{0}(h)>0$,

$$
v(h, n)=v(h, n+1) \quad \text { for all } n \in \mathbb{N} \text {. }
$$

We might as well drop the time subscripts and write $v(h)$ for $v(h, n)$, etc., where there is no risk of confusion. From Proposition 3 itself we know that for any $i \in I$ and $j \in J$ such that $\mu_{0}(i)>0$ and $\mu_{0}(j)>0$,

$$
v(i)+v(j) \geqslant s_{i}-t_{j}
$$

In order to prove the converse of (3) it is necessary to prove a stronger result, namely, that for each $\alpha \in A$, the value functions $v_{\alpha}$ are unique and, in an appropriate sense, stationary.

If the relation described by Eq. (1) is assumed to hold for every $\alpha \in A$ then for any $n \in \mathbb{N}$ the probability of meeting an agent of type $h \in H$ at date 
$n$ is independent of $\alpha$ and $n$. We can write, without loss of generality, $p_{\alpha}(h, n)=p(h)$, for any $h \in H, n \in \mathbb{N}$ and $\alpha \in A$. The recursive equations in Proposition 1 can be restated as

$$
\begin{aligned}
v_{\alpha}(i, n)= & \left(1-\frac{1}{2} \sum_{i \in J} p(j)\right) v_{\alpha}(i, n+1) \\
& +\frac{1}{2} \sum_{j \in J} p(j) \max \left\{\alpha^{n}\left(s_{i}-t_{j}\right)-v_{\alpha}(j, n+1), v_{\alpha}(i, n+1)\right\}
\end{aligned}
$$

for $n \in \mathbb{N}$ and $i \in I$ and

$$
\begin{aligned}
v_{\alpha}(j, n)= & \left(1-\frac{1}{2} \sum_{i \in I} p(i)\right) v_{\alpha}(j, n+1) \\
& +\frac{1}{2} \sum_{i \in I} p(i) \max \left\{\alpha^{n}\left(s_{i}-t_{j}\right)-v_{\alpha}(i, n+1), v_{\alpha}(j, n+1)\right\}
\end{aligned}
$$

for $n \in \mathbb{N}$ and $j \in J$.

Proposition 9. For any $\alpha \in A$ there exists a unique value function $v_{x}$ which is stationary in the sense that $v_{x}(h, n+1)=\alpha v_{\alpha}(h, n)$ for every $h \in H$ and $n \in \mathbb{N}$.

Proof. For any $h \in H$ and $n \in \mathbb{N}$, let $M_{\alpha}(h, n)$ denote the supremum of $v_{\alpha}(h, n)$ taken over all functions $v_{\alpha}$ satisfying equations (4) and (5) and the feasibility condition $0 \leqslant v_{x}(h, n) \leqslant \alpha^{n}\left(s_{1}-t_{1}\right)$ for the given matching probabilities $p$. Similarly for any $h \in H$ and $n \in \mathbb{N}$ let $m_{\alpha}(h, n)$ denote the infimum of $v_{\alpha}(h, n)$ taken over all functions $v_{\alpha}$ satisfying (4) and (5) and the feasibility condition. It is clear from inspection of (4) and (5) that $M_{\alpha}(h, n+1)=\alpha M_{\alpha}(h, n)$ and $m_{\alpha}(h, n+1)=\alpha m_{\alpha}(h, n)$. Thus, (4) implies that for any $i \in I$ and $n \in \mathbb{N}$,

$$
\begin{aligned}
m_{\alpha}(i, n) \geqslant & \left(1-\frac{1}{2} \sum_{j \in J} p(j)\right) m_{\alpha}(i, n+1) \\
& +\frac{1}{2} \sum_{j \in J} p(j) \max \left\{\alpha^{n}\left(s_{i}-t_{j}\right)-M_{\alpha}(j, n+1), m_{\alpha}(i, n+1)\right\} \\
= & \left(1-\frac{1}{2} \sum_{j \in J} p(j)\right) \alpha m_{\alpha}(i, n) \\
& +\frac{1}{2} \sum_{j \in J} \max \left\{\alpha^{n}\left(s_{i}-t_{j}\right)-\alpha M_{\alpha}(j, n), \alpha m_{\alpha}(i, n)\right\}
\end{aligned}
$$


and

$$
\begin{aligned}
M_{\alpha}(i, n) \leqslant & \left(1-\frac{1}{2} \sum_{j \in J} p(j)\right) M_{\alpha}(i, n+1) \\
& +\frac{1}{2} \sum_{j \in J} p(j) \max \left\{\alpha^{n}\left(s_{i}-t_{j}\right)-m_{\alpha}(j, n+1), M_{\alpha}(i, n+1)\right\} \\
= & \left(1-\frac{1}{2} \sum_{j \in J} p(j)\right) \alpha M_{\alpha}(i, n) \\
& +\frac{1}{2} \sum_{j \in J} p(j) \max \left\{\alpha^{n}\left(s_{i}-t_{j}\right)-\alpha m_{\alpha}(j, n), \alpha M_{\alpha}(i, n)\right\} .
\end{aligned}
$$

Subtracting (6) from (7) and rearranging yields

$$
\begin{aligned}
{[1-\alpha} & \left.\left(1-\frac{1}{2} \sum_{j \in J} p(j)\right)\right]\left(M_{\alpha}(i, n)-m_{\alpha}(i, n)\right) \\
\leqslant & \frac{1}{2} \sum_{j \in J} p(j) \max \left\{\alpha^{n}\left(s_{i}-t_{j}\right)-\alpha m_{\alpha}(j, n), \alpha M_{\alpha}(i, n)\right\} \\
& \quad-\frac{1}{2} \sum_{j \in J} p(j) \max \left\{\alpha^{n}\left(s_{i}-t_{j}\right)-\alpha M_{\alpha}(j, n), \alpha m_{\alpha}(i, n)\right\} \\
\leqslant & \frac{1}{2} \sum_{j \in J} p(j) \max \left\{\alpha\left[M_{\alpha}(j, n)-m_{\alpha}(j, n)\right], \alpha\left[M_{\alpha}(i, n)-m_{\alpha}(i, n)\right]\right\} .
\end{aligned}
$$

By analogous reasoning a similar inequality is derived for $j \in J$ and any $n \in \mathbb{N}$. Suppose that for some $i \in I$

$$
M_{\alpha}(i, n)-m_{\alpha}(i, n)=\max _{h \in H}\left\{M_{\alpha}(h, n)-m_{\alpha}(h, n)\right\} .
$$

Then the preceding inequality (8) implies that

$$
\begin{aligned}
& M_{\alpha}(i, n)-m_{\alpha}(i, n) \\
& \leqslant\left[1-\alpha\left(1-\frac{1}{2} \sum_{j \in J} p(j)\right)\right]^{-1} \alpha \frac{1}{2} \sum_{j \in J} p(j)\left[M_{\alpha}(i, n)-m_{\alpha}(i, n)\right],
\end{aligned}
$$

which implies that $M_{\alpha}(i, n)=m_{\alpha}(i, n)$ since, by definition, $M_{\alpha}(i, n) \geqslant$ $m_{\alpha}(i, n)$, both are finite and

$$
\frac{(1 / 2) \sum p(j) \alpha}{1-\alpha\left(1-(1 / 2) \sum p(j)\right)}<1 .
$$

A similar argument works for the case where some $j \in J$ maximizes $M_{\alpha}(h, n)-m_{\alpha}(h, n)$. Uniqueness of $v_{\alpha}$ is immediate because $m_{\alpha}(h, n) \leqslant$ 
$v_{\alpha}(h, n) \leqslant M_{\alpha}(h, n)$ and the stationarity property follows from $M_{\alpha}(h, n+1)=\alpha M_{\alpha}(h, n)$.

Proposition 10. For any $i \in I$ and $j \in J$ such that $v(i)>0$ and $v(j)>0$, $v(i)+v(j)=s_{i}-t_{j}$.

Proof. Suppose that for some $i \in I$ and all $j \in J, v(i)+v(j)>s_{i}-t_{j}$. Then for any $n \in \mathbb{N}$ and $\alpha$ sufficiently large,

$$
v_{\alpha}(i, n+1)+v_{\alpha}(j, n+1)>\alpha^{n}\left(s_{i}-t_{j}\right) \quad \text { for all } j \in J .
$$

By stationarity this means that $i$ is unable to trade with any $j \in J$ at any date $n \in \mathbb{N}$. But this means $v_{\alpha}(i, n+1)=0$, and in the limit $v(i)=0$. Thus for any $i \in I$ such that $v(i)>0$ there exists $j \in J$ such that $v(i)+v(j) \leqslant s_{i}-t_{j}$. Then $\psi(i, j, n)=p(j)>0$ for all $n \in \mathbb{N}$ and this implies $\phi(i, \infty)=0$. By the argument used in Section $5, \phi(i-1, \infty)=0$ implics that $\Delta_{i} v(i)=\Delta_{i} s_{i}$. An exactly similar argument shows that if $v(j+1)>0$ then $\Delta_{i} v(j)=-\Delta_{j} t_{j}$.

Let $i_{0}$ (resp. $j_{0}$ ) be the largest value of $i \in I$ (resp. $j \in J$ ) such that $v(i)>0$ (rcsp. $v(j)>0$ ). We know that $v\left(i_{0}\right)+v\left(j_{0}\right) \geqslant s_{i 0}-t_{j 0}$. Since $\Delta_{i} v(i)=A_{i} s_{i}$ for $i<i_{0}$ and $\Delta_{j} v(j)=-\Delta_{j} t_{j}$ for $j<j_{0}$, if the inequality were strict it would imply that $v(i)+v(j)>s_{i}-t_{j}$ for all $i \in I$ and $j \in J$, a contradiction. Thus, $v\left(i_{0}\right)+v\left(j_{0}\right)=s_{i_{0}}-t_{j_{0}}$ and so $v(i)+v(j)-s_{i}-t_{j}$ for all $(i, j) \leqslant\left(i_{0}, j_{0}\right)$ as claimed.

It is immediate from Proposition 10 that there exists a "price" $\omega \geqslant 0$ such that for any $i \in I$,

$$
v(i)=\max \left\{s_{i}-\omega, 0\right\}
$$

and for any $j \in J$,

$$
v(j)=\max \left\{\omega-t_{j}, 0\right\} .
$$

Typically the situation will be as described in Fig. 1 where the marginal trading types on each side of the market receive positive utility. Generically, this is not a Walrasian equilibrium relative to $\mu_{0}$ because demand does not equal supply. Still the fact that there is a single uniform price at which all trades take place is important in its own right. It confirms the claim made by RW in [13] that their model provides a theory of price formation. This claim could not be tested in the context of their original example because it was too rudimentary to discriminate between price and non-price outcomes.

Proposition 10 only tells us that a price exists at which all trades take place. It does not tell us how the price is determined. The next proposition cnables us to derive the price $\omega$ from knowledge of the steady-statc distribution $\mu_{0}$. 
Proposition 11. $\sum_{i \in I} p(i) v(i)=\sum_{j \in J} p(j) v(j)$.

Proof. From Proposition 10 and the recursive equations (4) and (5) we know that for any $\alpha \in A$ and $i \in I$,

$$
(1-\alpha) v_{\alpha}(i, 0)=\frac{1}{2} \sum_{j \in J} p(j) \alpha \max \left\{s_{i}-t_{j}-v_{\alpha}(i, 0)-v_{\alpha}(j, 0), 0\right\} .
$$

Multiplying both sides by $p(i)$ and summing over $i \in I$ gives

$$
\begin{aligned}
\sum_{i \in I}(1-\alpha) p(i) v_{\alpha}(i, 0) & \\
& =\frac{1}{2} \sum_{i \in I} \sum_{j \in J} p(i) p(j) \alpha \max \left\{s_{i}-t_{j}-v_{\alpha}(i, 0)-v_{\alpha}(j, 0), 0\right\} .
\end{aligned}
$$

A symmetrical argument shows that

$$
\begin{aligned}
& \sum_{j \in J}(1-\alpha) p(j) v_{\alpha}(j, 0) \\
& \quad=\frac{1}{2} \sum_{i \in I, j \in J} \sum_{j \in J} p(i) p(j) \alpha \max \left\{s_{i}-t_{j}-v_{\alpha}(i, 0)-v_{\alpha}(j, 0), 0\right\} .
\end{aligned}
$$

Since $\alpha<1$,

$$
\sum_{i \in I} p(i) v_{\alpha}(i, 0)=\sum_{j \in J} p(j) v_{x}(j, 0)
$$

for any $\alpha \in A$. Taking limits as $\alpha \rightarrow 1$ gives the required result.

Roughly speaking this proposition tells us that the sums of the expected utilities on each side of the market at a given date are equal. This is, of course, a consequence of assuming that all agents have the same rate of time preference. That assumption, which is innocuous in other respects, is crucial here. If, for example, sellers were more impatient than buyers the sum of their expected utilities at a given date would be smaller than the buyers'. And by varying their relative discount factors we could generate any desired distribution of surplus between the two sides of the market. Proposition 11 is the analogue of the result obtained by RW in [13]. Like their result it seems inconsistent with Walrasian equilibrium. To be precise, the steady state distribution $\mu_{0}$ defines an exchange economy which has a unique Walrasian equilibrium price. There is no reason to think that that price is the same as the value of $\omega$ determined by Proposition 11. And if by chance it were, varying the relative discount rates would vary $\omega$ without altering the "Walrasian" price. In short, $\omega$ is not except by sheer coincidence a Walrasian price in this sense. 
More formally, let us write $v \in W\left(\mu_{0}\right)$ if there exists a price $\omega \geqslant 0$ such that $v(i)=\max \left\{s_{i}-\omega, 0\right\}$ for $i \in I$ and $v(j)=\max \left\{\omega-t_{j}, 0\right\}$ for $j \in J$ and the following market-clearing condition is satisfied: either $\sum_{i=1}^{i_{0}-1} \mu_{0}(i) \leqslant$ $\sum_{j=1}^{j_{0}} \mu_{0}(j) \leqslant \sum_{i=1}^{i_{0}} \mu_{0}(i)$ with $v\left(i_{0}\right)=e$ if the last inequality is strict or $\sum_{j=1}^{j_{0}-1} \mu_{0}(j) \leqslant \sum_{i=1}^{i_{0}} \mu_{0}(i) \leqslant \sum_{j=1}^{j_{0}} \mu_{0}(j)$ with $v\left(j_{0}\right)=e$ if the last inequality is strict, where $i_{0}=\max \{i \in I \mid v(i) \geqslant e\}$ and $j_{0}=\max \{j \in J \mid v(j) \geqslant e\}$. If $v \in W\left(\mu_{0}\right)$ we say that $v$ is Walrasian with respect to $\mu_{0}$. The formal conclusion of the preceding discussion is the following theorem.

THEOREM 2. Let $\left(v_{\alpha}, \mu_{\alpha}\right)$ be a sequence of perfect equilibria for any economy with a steady-state distribution of agents $\mu_{x}(\cdot, n)=\mu_{0}$ for all $\alpha$ and $n$. If $(v, \mu)$ is a limit point of this sequence then for some values of $\mu_{0}$, $v \notin W\left(\mu_{0}\right)$ because the market clearing conditions are violated.

Onc can also define a flow equilibrium. In this particular case any price is market-clearing in the flow sense since by assumption buyers and sellers flow into and out of the market in equal numbers. And this is exactly what is required since, as we have seen, any price can be generated by altering the relative rates of time preference. In this example, the flow equilibrium concept seems somewhat unhelpful. The price $\omega$ is determined by the stock of agents $\mu_{0}$ together with the condition in Proposition 11, whereas the flow market-clearing condition determines nothing. Indeed it cannot determine anything since it is always trivially satisfied. However, these properties of the model seem to arise from the fact that, by assumption, the flows into the market are always balanced and hence the stock of agents in the market is effectively exogenous.

To test this hypothesis, consider a different set of entry assumptions. Suppose that at each date $n \in \mathbb{N}$ there is a constant stream of potential entrants represented by the measure $v_{0}$. In terms of the earlier notation, for each $n \in \mathbb{N}$,

$$
v(\cdot, n)=v_{0} .
$$

It is clearly impossible to have a steady state unless agents who cannot trade are excluded from the market. Assume there is a small entry fee $e>0$. Then agents must choose whether or not to enter the market. As before, consider a sequence $\left\{\left(v_{0}, \mu_{\alpha}\right)\right\}_{\alpha \in A}$ of perfect equilibria with limit $(v, \mu)$ and suppose that for each $\alpha \in A$, the matching probabilities $p_{x}$ are timeinvariant, that is,

$$
p_{x}(\cdot, n)=p_{x}(\cdot, n+1) \quad \text { for all } n \in \mathbb{N} .
$$

Clearly (10) implies the same property holds in the limit as $\alpha \rightarrow 1$.

Propositions 9, 10, and 11 continue to hold under the revised entry assumption (9) since the only property of $\mu_{x}$ used in the proofs is 
stationarity, which continues to hold by assumption. Now, however, an additional condition must be satisfied. Since buyers and sellers leave the market in equal numbers they must enter in equal numbers to maintain a stationary equilibrium. This is the flow market-clearing condition and it implies conditions analogous to (5.15) and (5.16). Let $i_{0}=$ $\max \left\{i \in I \mid s_{i} \geqslant \omega+e\right\}$ and $j_{0}=\max \left\{j \in J \mid t_{j} \leqslant \omega-e\right\}$. Then one of the following must hold.

Either

$$
\begin{aligned}
\sum_{i=1}^{i_{0}-1} v_{0}(i) \leqslant \sum_{j=1}^{j_{0}} v_{0}(j) \leqslant \sum_{i=1}^{i_{0}} \nu 0(i), \\
\text { with } s_{i_{0}}=\omega+e \text { if the last inequality is strict, }
\end{aligned}
$$

or

$$
\begin{aligned}
\sum_{j=1}^{j_{0}-1} v_{0}(j) \leqslant \sum_{i=1}^{i_{0}} v_{0}(i) \leqslant \sum_{j=1}^{j_{0}} v_{0}(j) \\
\text { with } t_{j_{0}}=\omega-e \text { if the last inequality is strict. }
\end{aligned}
$$

The proof is similar to the one given at the end of Section 5 and will not be repeated here.

THEOREM 3. Let $\left(v_{\alpha}, \mu_{x}\right)$ be a sequence of perfect equilibria for an economy with a constant stream of potential entrants $v(\cdot, n)=v_{0}$ for all $n$ and a small entry fee $e>0$. If $\mu_{\alpha}(\cdot, n)=\mu_{0}$ for all $\alpha$ and $n$ and $(v, \mu)$ is a limit point of the sequence then $v$ is Walrasian in the sense that $v \in W\left(v_{0}\right)$.

Conditions (11) and (12) generically define a unique price $\omega$ which in turn determines everything of interest about the limit point $(v, \mu)$. In other words the data in $v_{0}$ determine the limit of the perfect equilibria. On the other hand, the steady state distribution $\mu$ determines nothing of interest. Proposition 11 continues to hold but it tells us nothing. The payoffs to individual agents are determined independently of the condition in Proposition 11 and it is satisfied entirely by adjustments in $\mu$. What then is the relationship between $\mu, v_{0}$, and $\omega$ ? It follows directly from the steadystate assumption and the matching rules that the distribution of entrants at each date must be proportional to the distribution of agents in the market. More precisely, there exists scalars $\lambda_{1}$ and $\lambda_{2}$ such that

$$
\begin{array}{ll}
\lambda_{1} \mu(i, n)=v_{0}(i) & \left(i<i_{0}\right) \\
\lambda_{2} \mu(j, n)=v_{0}(j) & \left(j<j_{0}\right) .
\end{array}
$$


In the case of the marginal types $i_{0}$ and $j_{0}$,

$$
\begin{aligned}
& \lambda_{1} \mu\left(i_{0}, n\right)=\min \left\{\sum_{i=1}^{i_{0}} v_{0}(i), \sum_{j=1}^{j_{0}} v_{0}(j)\right\}-\sum_{i=1}^{i_{0}-1} v_{0}(i) \\
& \lambda_{2} \mu\left(j_{0}, n\right)=\min \left\{\sum_{i=1}^{i_{0}} v_{0}(i), \sum_{j=1}^{j_{0}} v_{0}(j)\right\}-\sum_{j=1}^{j_{0}-1} v_{0}(j) .
\end{aligned}
$$

Obviously $\mu(i, n)=0=\mu(j, n)$ for $i>i_{0}$ and $j>j_{0}$. To ensure that $\mu$ is time invariant the measure of departing agents of each type must equal the measure of entering agents. For example, the number of departing agents of type $i=1$ is $\mu(i=1, n) p(J)$. (This requires separate proof that all pairs of agents $(i, j)=(1, j)$ who meet actually trade). So assuming $i_{0}>1$, we have

$$
v_{0}(i=1)=\mu(i=1, n) p(J)=\mu(i=1, n) \mu(J, n) / \mu(H, n) .
$$

Substituting from (13) into (17) we see that

$$
\lambda_{1}=\mu(J, n) / \mu(H, n) .
$$

A similar argument shows that

$$
\lambda_{2}=\mu(I, n) / \mu(H, n) .
$$

Then (18) and (19) imply $\lambda_{1}+\lambda_{2}=1$. Thus $\mu$ is determined, up to a scalar, by $(13-16)$ in terms of $v_{0}$ and $\omega$. The scalar which remains to be determined $\left(\lambda_{1} / \lambda_{2}\right)$ is fixed by Proposition 11 . The ratio of buyers to sellers is adjusted so that the sums of expected utilities on the two sides of the market are equal. In this precise sense Proposition 12 has content: it only determines the size of the queue and has no effect on individual payoffs.

To sum up, under the entry assumption (9) the limiting price $\omega$ is determined by $v_{0}$, the flow of potential entrants, and by the flow market-clearing conditions (11) and (12). The stock of agents in the market $\mu$ is determined up to a scalar by $v_{0}$ and $\omega$. Nothing of interest depends on $\mu$ in the limiting economy.

These formal results, taken together with those for non-steady states, argue strongly that the flow concept of market-clearing is the appropriate one. It is the rather special properties of the RW model which make the stock concept seem more appropriate there and hence generate the paradox. 


\section{REFERENCES}

1. K. Binmore and M. Herrero, "Matching and Bargaining in Dynamic Markets I," mimeo, London School of Economics, 1985.

2. K. Binmore AND M. Herrero, "Matching and Bargaining in Dynamic Markets II," mimeo, London School of Economics, 1985.

3. P. Diamond, Mobility costs, frictional unemployment and efficiency, J. Polit. Econ. 89 (1981), 798-812.

4. P. Diamond, Wage determination and efliciency in search equilibrium, Rev. Econ. Stud. 49 (1982), 217-227.

5. D. Gale, "Equilibrium in a Market with Sequential Bargaining and No Transaction Costs is Walrasian," ICERD Discussion Paper No. 84/104, London School of Economics, 1984.

6. D. Gale, "Bargaining and Competition," CARESS Working Paper No. 84/23, University of Pennsylvania, 1984.

7. D. GALE, "Decentralized Exchange and Existence of Perfect Equilibrium in a Class of Bargaining Games," CARESS Working Paper 85/04, University of Pennsylvania, 1985.

8. M. MANDEL, "Are Search-Type Models a Good Approximation to Competitive Labor Markets?" mimeo, Harvard University, 1985.

9. D. MORTENSEN, Property rights and efficiency in mating, racing and related games, Amer. Econ. Rev. 72 (1982), 968-979.

10. D. MorTENSEN, The matching process as a noncooperative bargaining game, Chap. 7, in "The Economics of Information and Uncertainty" (J. McCall, Ed.), Univ. of Chicago Press, Chicago, 1982.

11. A. Rubinstein, Perfect equilibrium in a bargaining model, Econometrica 50 (1982), 97-109.

12. A. Rubinstein, "A Sequential Strategic Theory of Bargaining," Paper presented to World Congress of the Econometric Society, Cambridge, MA, 1985.

13. A. RUBinstein AND A. WolinsKy, Equilibrium in a market with sequential bargaining, Econometrica 53 (1985), 1133-1150.

14. A. Shaked AND J. SitTON, Involuntary unemployment as a perfect equilibrium in a bargaining model, Econometrica 52 (1984), 1351-1364.

15. A. Shaked AND J. Sutron, "The Semi-Walrasian Economy," ICERD Discussion Paper No. 84/98, London School of Economics, 1984. 